\title{
Holographic oddballs
}

\section{Bellantuono, ${ }^{a, b}$ P. Colangelo ${ }^{b}$ and F. Giannuzzi ${ }^{a}$}

${ }^{a}$ Dipartimento di Fisica, Università di Bari, via Orabona 4, I-70126 Bari, Italy

${ }^{b}$ INFN - Sezione di Bari, via Orabona 4, I-70126 Bari, Italy

E-mail: loredana.bellantuono@ba.infn.it, pietro.colangelo@ba.infn.it, floriana.giannuzzi@ba.infn.it

ABSTRACT: The spectrum of the glueball with $J^{P C}=0^{--}$is computed using different bottom-up holographic models of QCD. The results indicate a lowest-lying state lighter than in the determination by other methods, with mass $m \simeq 2.8 \mathrm{GeV}$. The in-medium properties of this gluonium are investigated, and stability against thermal and density effects is compared to other hadronic systems. Production and decay modes are identified, useful for searching the $J^{P C}=0^{--}$glueball.

KEYWORDS: QCD Phenomenology, Strings and branes phenomenology

ARXiv EPRINT: 1507.07768 


\section{Contents}

1 Introduction 1

2 Oddballs in bottom-up AdS/QCD 2

2.1 Hard-wall model (HW) 3

2.2 Soft-wall model (SW) 4

2.3 Einstein-dilaton model (ED) 5

3 Oddballs in medium $\quad 6$

$\begin{array}{lll}4 J^{\mathrm{PC}}=0^{--} \text {glueball phenomenology } & 11\end{array}$

5 Conclusions 13

\section{Introduction}

The existence of bound states of gluons (the so-called "gluonia" or "glueballs"), with a rich spectroscopy and a complex phenomenology, is one of the early predictions resulting from the non-Abelian nature of strong interactions described by QCD [1]. However, after about 40 years of experimental efforts, evidence of no one of such gluonic states has been unambiguously established [2]. The glueball with $J^{\mathrm{PC}}=0^{++}$, expected to be the lightest one, shares the vacuum quantum numbers with $\bar{q} q$ states; as a consequence, hadrons can result from the mixing between quark-antiquark and gluonium components. The scalar isoscalar mesons $f_{0}(1370), f_{0}(1500)$ and $f_{0}(1710)$ are candidates for such light mixed states, with uncertain mixing angles inferred from the production processes and decay modes [3]. The $J^{\mathrm{PC}}=2^{++}$glueball, predicted to be heavier than the scalar one, can mix with conventional $\bar{q} q P$-wave configurations, therefore also the identification of the tensor glueball is difficult. A signature for the experimental investigations is that gluonia overpopulate the meson multiplets with fixed quantum numbers. Moreover, since glueballs are $\mathrm{SU}(3)_{F}$ singlets, they are expected to equally couple to $u, d$ and $s$ quarks with clear predictions of the decay fractions in pions, etas and kaons. However, the chiral behaviour of such couplings needs also to be taken into account: it can induce deviations from the flavor-symmetric condition due to a quark mass dependence, as discussed for the $J^{\mathrm{PC}}=0^{++}$state in [4], and this makes the glueball identification further involved.

Remarkably, there is the possibility of having gluonia with combinations of $J^{\mathrm{PC}}$ not allowed in the quark model; therefore, searching for states with such "exotic" quantum numbers is a strategy to look for gluonic resonances. An interesting case is $J^{\mathrm{PC}}=0^{--}$: glueballs with these quantum numbers, as for all states with negative charge conjugation, must be composed by an odd number of constituent gluons, which justifies the name of "oddballs". Although $C$-odd gluonia are expected to be heavier than the scalar glueball, they are within the reach of the present-day experimental facilities. 
Little theoretical information is available about gluonic resonances with $J^{\mathrm{PC}}=0^{--}$. In the old flux-tube model, Isgur and Paton predicted $m_{0^{--}}=2.79 \mathrm{GeV}$, with the mass ordering in the gluonium spectrum: $m_{0^{++}}<m_{0^{--}} \sim m_{2^{++}}$[5]. Lattice QCD simulations have reported a large value for the mass: $m_{0^{--}}=5.166 \mathrm{GeV}$, with an estimated uncertainty of $1 \mathrm{GeV}$; in the same calculation, the mass of the lightest glueball is $m_{0^{++}}=1.795 \mathrm{GeV}$, with $3.3 \%$ uncertainty [6]. Two stable $0^{--}$oddballs have been obtained using QCD sum rules, with mass $m_{0^{--}}=3.81 \pm 0.12 \mathrm{GeV}$ and $m_{0^{--}}=4.33 \pm 0.13 \mathrm{GeV}$, respectively [7]. In all cases, the width and the hadronic couplings are unknown. On the experimental side, analyses of the $D^{0} \rightarrow \pi^{+} \pi^{-} \pi^{0}$ decay mode indicate that the final state is nearly completely dominated by a $I=0, J^{\mathrm{PC}}=0^{--}$configuration [8]; however, the confirmation of the contribution of a resonance close to $D^{0}$ (the $D^{0}$ mass is $1864.84 \mathrm{MeV}$ ), together with the interpretation of this puzzling result, requires a further scrutiny.

An interesting method for computing various hadronic properties is inspired by the AdS/CFT (Anti-de Sitter/conformal field theory) correspondence [9-11]. In this approach, at large 't Hooft coupling and in the limit of large number of colors $N_{c}$, the correlation functions of gauge-invariant operators in a $4 D$ gauge field theory are derived by a classical gravity theory in a higher dimensional space. ${ }^{1}$ The calculation of the mass of the scalar $J^{\mathrm{PC}}=0^{++}$and tensor $J^{\mathrm{PC}}=2^{++}$glueballs is one of the first applications of the method [13-18], starting from the top-down construction based on a type II-A supergravity with supersymmetry and conformal invariance broken, and a Yang-Mills theory at large $N_{c}$ as a dual [10]. Including matter fields, the analysis has been extended to the glueball hadronic couplings $[19,20]$. The mass spectrum of the $C$-odd $J^{\mathrm{PC}}=1^{+-}$and $1^{--}$ glueballs has been computed in the top-down construction, too [17].

The gauge/gravity duality method has also been applied in a more phenomenological procedure which attempts to formulate, through a bottom-up construction, higher dimensional models able to reproduce the largest number of QCD properties. Here, we follow this bottom-up approach which has been used to describe conventional $\bar{q} q[21-25]$ and hybrid mesons [26], as well as glueballs [27-30]. We focus on the lightest oddball with the aim of determining properties like the mass spectrum.

In section 2 we compute the spectrum of the $J^{\mathrm{PC}}=0^{--}$gluonium in three different holographic models of QCD, identifying robust predictions and discussing the uncertainties. Other interesting aspects to investigate concern the properties of the glueball in a thermalized and dense hadronic medium. Pointing out the differences with respect to ordinary $\bar{q} q$ mesons provides us with a better understanding of this gluonium state, namely about its stability features. The calculation is affordable in the holographic framework, and is carried out in section 3. From the experimental viewpoint, it is important to identify the main production processes and the decay modes useful for the searches of the $J^{\mathrm{PC}}=0^{--}$ glueball: this is done in section 4, before presenting the conclusions of our study.

\section{Oddballs in bottom-up AdS/QCD}

In gauge/gravity inspired models of QCD, the starting point is the association of gauge invariant boundary theory operators to fields defined on an $A d S_{5}$ manifold, with modifica-

\footnotetext{
${ }^{1}$ An overview can be found in [12].
} 
tions needed to describe confinement. In QCD, composite gauge-invariant local operators with quantum numbers $J^{\mathrm{PC}}=0^{--}$, involving only gluon fields, can be written in terms of the gluon field strength $G_{\mu \nu}^{a}(x)$ and the dual $\widetilde{G}_{\mu \nu}^{a}(x)=\frac{1}{2} \epsilon_{\mu \nu \rho \sigma} G_{\rho \sigma}^{a}(x)$ :

$$
\begin{aligned}
& J^{A}(x)=g_{s}^{3} d_{a b c}\left[\eta_{\alpha \beta}^{t} \widetilde{G}_{\mu \nu}^{a}(x)\right]\left[\partial_{\alpha} \partial_{\beta} G_{\nu \rho}^{b}(x)\right]\left[G_{\rho \mu}^{c}(x)\right] \\
& J^{B}(x)=g_{s}^{3} d_{a b c}\left[\eta_{\alpha \beta}^{t} G_{\mu \nu}^{a}(x)\right]\left[\partial_{\alpha} \partial_{\beta} \widetilde{G}_{\nu \rho}^{b}(x)\right]\left[G_{\rho \mu}^{c}(x)\right] \\
& J^{C}(x)=g_{s}^{3} d_{a b c}\left[\eta_{\alpha \beta}^{t} G_{\mu \nu}^{a}(x)\right]\left[\partial_{\alpha} \partial_{\beta} G_{\nu \rho}^{b}(x)\right]\left[\widetilde{G}_{\rho \mu}^{c}(x)\right] \\
& J^{D}(x)=g_{s}^{3} d_{a b c}\left[\eta_{\alpha \beta}^{t} \widetilde{G}_{\mu \nu}^{a}(x)\right]\left[\partial_{\alpha} \partial_{\beta} \widetilde{G}_{\nu \rho}^{b}(x)\right]\left[\widetilde{G}_{\rho \mu}^{c}(x)\right],
\end{aligned}
$$

with $a, b, c$ color indices, $d_{a b c}$ the symmetric $\mathrm{SU}(3)_{c}$ structure constants, and $g_{s}$ the strong coupling constant. The transverse $\eta_{\alpha \beta}^{t}$ metric is defined as $\eta_{\alpha \beta}^{t}=\eta_{\alpha \beta}-\frac{\partial_{\alpha} \partial_{\beta}}{\partial^{2}}$, with $\alpha, \beta$ (as well as $\mu, \nu) 4 D$ Lorentz indices, and $\eta_{\alpha \beta}$ the Minkowski metric tensor [7].

We focus on only one operator in (2.1), generically denoted as $J_{0}(x)$, which has conformal dimension $\Delta=8$, and associate to $J_{0}(x)$ a dual field in $A d S_{5}, O_{0}(x, z)$, with mass obtained by the relation $M_{5}^{2} R^{2}=\Delta(\Delta-4)[10,11] . R$ is the $A d S_{5}$ radius; we set $R=1$.

We choose Poincarè coordinates $x_{M}=\left(x_{\mu}, z\right)=\left(x_{0}, \vec{x}, z\right)$ for the $A d S_{5}$ space, with line element

$$
d s^{2}=g^{M N} d x_{M} d x_{N}=\frac{1}{z^{2}}\left(d x_{0}^{2}-d \vec{x}^{2}-d z^{2}\right)
$$

$(0<z)$, and define an action for the field $O_{0}(x, z)$. To account for confinement in QCD, in the definition of the action the breaking of conformal invariance must be implemented: this can be done in different ways, some of which are used in the following.

\subsection{Hard-wall model (HW)}

A simple way of modeling confinement in the holographic setup is by considering a slice of the $\mathrm{AdS}_{5}$ space, with a sharp cutoff at a finite distance $z_{m}$ along the extra dimension [21, 22]. The $5 D$ action for $O_{0}(x, z)$ can then be written as

$$
S_{(H W)}=\frac{1}{k} \int d^{5} x \sqrt{g}\left[g^{M N} \partial_{M} O_{0} \partial_{N} O_{0}-M_{5}^{2} O_{0}^{2}\right]
$$

with $\epsilon \leq z \leq z_{m}$ and $g=\left|\operatorname{det}\left(g_{M N}\right)\right|$. The constant $k$ makes the action dimensionless, and its derivation is presented in the next section. The value of $1 / z_{m}$ sets the hadronic scale in the model, so that the dimensionful quantities are given in terms of this parameter.

The equation of motion for the Fourier image $\widetilde{O}_{0}(p, z)$ of $O_{0}(x, z)$,

$$
-\frac{p^{2}}{z^{3}} \widetilde{O}_{0}(p, z)-\partial_{z}\left[\frac{1}{z^{3}} \partial_{z} \widetilde{O}_{0}(p, z)\right]+\frac{M_{5}^{2}}{z^{5}} \widetilde{O}_{0}(p, z)=0
$$

together with the Dirichlet boundary condition for $\widetilde{O}_{0}(p, z)$ at the ultraviolet $z=\epsilon$ brane, $\widetilde{O}_{0}(p, \epsilon)=0$ (with $\epsilon \rightarrow 0^{+}$), and the Neumann boundary condition at the infrared $z=z_{m}$ brane, $\partial_{z} \widetilde{O}_{0}\left(p, z_{m}\right)=0$, allows to compute the spectrum. The resulting lightest masses, setting $1 / z_{m}=346 \mathrm{MeV}$, are collected in figure 1 . The masses of the first two states are $m_{0}=2.80 \mathrm{GeV}$ and $m_{1}=4.14 \mathrm{GeV}$, respectively. Such values reduce to $m_{0}=2.61 \mathrm{GeV}$ and $m_{1}=3.86 \mathrm{GeV}$ if the hadronic scale is $1 / z_{m}=323 \mathrm{MeV}$; both values of $1 / z_{m}$ are used 


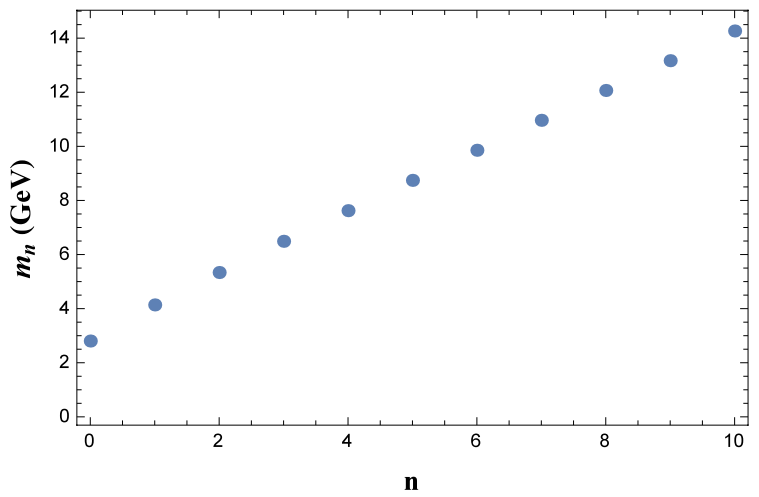

Figure 1. Spectrum of the $J^{\mathrm{PC}}=0^{--}$gluonium by the HW model, with $1 / z_{m}=346 \mathrm{MeV}$.

in phenomenological analyses [21]. A linear dependence is obtained for $m_{n}$ vs the radial (in the extra dimension) quantum number $n$, a feature shared by other hadrons described by this model.

\subsection{Soft-wall model (SW)}

A different way of breaking the conformal invariance and modeling confinement in QCD consists in including a background field $\phi(z)$ in the $5 D$ action for $O_{0}(x, z)$ [23]. In particular, the profile $\phi(z)=c^{2} z^{2}$, which involves a parameter $c$ fixing the hadronic scale, allows to obtain linear Regge trajectories and has been employed to study aspects of the light hadron phenomenology $[23,24]$. The action for the field $O_{0}(x, z)$ is

$$
S_{(S W)}=\frac{1}{k} \int d^{5} x \sqrt{g} e^{-\phi(z)}\left[g^{M N} \partial_{M} O_{0} \partial_{N} O_{0}-M_{5}^{2} O_{0}^{2}\right]
$$

with $\epsilon<z<\infty$. The normalizable solutions of the equation of motion for $\widetilde{O}_{0}(p, z)$,

$$
\frac{e^{-\phi(z)}}{z^{3}}\left(-p^{2}\right) \widetilde{O}_{0}(p, z)-\partial_{z}\left[\frac{e^{-\phi(z)}}{z^{3}} \partial_{z} \widetilde{O}_{0}(p, z)\right]+\frac{M_{5}^{2} e^{-\phi(z)}}{z^{5}} \widetilde{O}_{0}(p, z)=0,
$$

correspond to the Regge-like mass spectrum

$$
m_{n}^{2}=4 c^{2}(n+4)
$$

The gluonium turns out to be heavier than conventional $\bar{q} q$ mesons, than the $J^{P C}=1^{-+}$ hybrid and the $0^{++}$glueball, as one infers from the spectral relations in table 1 . However, the mass difference with respect to ordinary hadrons is smaller than obtained by different calculations. Setting $c=m_{\rho} / 2=388 \mathrm{MeV}$ from the $\rho$ meson mass [23], we get $m_{0}=$ $1.55 \mathrm{GeV}$ and $m_{1}=1.74 \mathrm{GeV}$, while the value $c=474 \mathrm{MeV}$ reproducing the $\rho$-meson Regge trajectory corresponds to $m_{0}=1.90 \mathrm{GeV}$ and $m_{1}=2.12 \mathrm{GeV}$. Hence, the SW model indicates a light oddball, as for other mass predictions in the same framework.

The two-point correlation function of $J_{0}(x)$ in QCD

$$
\Pi\left(p^{2}\right)=i \int d^{4} x e^{i p x}\left\langle 0\left|T\left[J_{0}(x) J_{0}^{\dagger}(0)\right]\right| 0\right\rangle
$$




\begin{tabular}{|cccccc|}
\hline$J^{\mathrm{PC}}$ & $1^{--}(q \bar{q})[23]$ & $0^{++}(q \bar{q})[24]$ & $0^{++}$(glueball) $[28]$ & $1^{-+}[26]$ & $0^{--}$ \\
\hline$m_{n}^{2}$ & $4 c^{2}(n+1)$ & $4 c^{2}(n+3 / 2)$ & $4 c^{2}(n+2)$ & $4 c^{2}(n+2)$ & $4 c^{2}(n+4)$ \\
\hline
\end{tabular}

Table 1. Mass spectrum of $q \bar{q}$ vector and scalar states, $0^{++}$glueball, $\bar{q} G q 1^{-+}$hybrid, and $0^{--}$ gluonium, obtained in the SW model.

can be computed using the AdS/CFT dictionary identifying $J_{0}(x)$ as the source of $O_{0}(x, z)$. We define the (4D Fourier transformed) bulk-to-boundary propagator $\widetilde{K}(p, z)$ of the glueball field using the equation:

$$
\widetilde{O}_{0}(p, z)=\widetilde{K}(p, z) \tilde{J}_{0}(p)
$$

with $\widetilde{O}_{0}(p, z)$ and $\widetilde{J}_{0}(p)$ the $4 \mathrm{D}$ Fourier transformed bulk field and source, respectively, and differentiate twice the on-shell action (2.5) with respect to $J_{0}$. The two-point correlation function, obtained for $J_{0} \rightarrow 0$,

$$
\Pi\left(p^{2}\right)=\frac{2}{k}\left[\frac{e^{-\phi(z)}}{z^{3}} \widetilde{K}\left(p^{2}, z\right) \partial_{z} \widetilde{K}\left(p^{2}, z\right)\right]_{z \rightarrow 0},
$$

can be written as

$$
\Pi\left(p^{2}\right)=\sum_{n} \frac{R_{n}}{p^{2}-m_{n}^{2}}
$$

with the residues

$$
R_{n}=-\frac{4}{15} \frac{(n+6) !}{6 ! n !} \frac{c^{14}}{k} .
$$

Comparing the $p^{2} \rightarrow-\infty$ asymptotic behaviour of $\Pi\left(p^{2}\right)$ in QCD [7]

$$
\Pi^{Q C D}\left(p^{2}\right)=\frac{487 \alpha_{s}^{3}}{1432^{6} 3^{3} \pi}\left(-p^{2}\right)^{6} \log \left(\frac{-p^{2}}{\Lambda^{2}}\right)
$$

with the expression (2.10) in the same limit, we fix $k$ (for $N_{c}=3$ ):

$$
k=-\frac{143 \pi}{4872^{10} 5^{2} \alpha_{s}^{3}} .
$$

The same expression holds in the HW model.

\subsection{Einstein-dilaton model (ED)}

In both the HW and SW models the implementation of the confinement mechanism, with an $A d S_{5}$ background geometry, is an input assumption defining each model. More elaborated approaches include dynamical fields. A dynamical holographic model of QCD has been formulated in [31, 32], with a scalar dilaton field $\Phi(z)$ in the bulk and the 5D gravitationdilaton coupled action analysed. The resulting geometry takes the form:

$$
d s_{(E D)}^{2}=\frac{e^{2 A_{s}(z)-\frac{4}{3} \Phi(z)}}{z^{2}}\left[d x_{0}^{2}-d \vec{x}^{2}-d z^{2}\right]
$$


The function $A_{s}(z)$ introduces a quadratic correction in the warp factor distorting the $A d S_{5}$ metric, and is chosen with the expression $A_{s}(z)=\bar{c} \bar{k}^{2} z^{2}$. The profile of the dilaton is obtained solving the Einstein equations for the metric-dilaton system, and with the chosen ansatz for $A_{s}(z), \Phi(z)$ reads

$$
\Phi(z)=\frac{3}{4} \bar{c} \bar{k}^{2} z^{2}\left(1+{ }_{2} F_{2}\left(1,1 ; 2, \frac{5}{2} ; 2 \bar{c} \bar{k}^{2} z^{2}\right)\right)
$$

in terms of the generalized hypergeometric function ${ }_{2} F_{2}$. In the model, the dimensionful parameter $\bar{k}$ is set to $\bar{k}=0.43 \mathrm{GeV} . \bar{c}$ is $\bar{c}= \pm 1$, and both cases reproduce, at finite temperature, QCD bulk thermodynamical properties such as the energy density and the speed of sound. On the other hand, the analysis of the thermodynamical properties of loop operators favours the positive sign; hence, we set $\bar{c}=+1$.

The equation of motion for the oddball field $O_{0}(x, z)$ with the metric (2.15)-(2.16) provides for the two lightest states the mass $m_{0}=2.82 \mathrm{GeV}$ and $m_{1}=4.07 \mathrm{GeV}$, respectively, close to the result of the HW model.

The outcome of the analyses in the three models is that the mass of the lowest-lying $0^{--}$state is sensibly lighter than obtained in $[6,7]$, with results spanning the range $1.55-2.82 \mathrm{GeV}$. The upper value, obtained in the HW and ED models, is close to the prediction of the flux-tube model. For the first excited state, the predicted mass is in the range $1.74-4.07 \mathrm{GeV}$, with again the lighter value given by the SW model. The indication in favour of a light oddball is a surprising result with interesting phenomenological implications. Indeed, one can look for this state in the same class of processes investigated for searching the $0^{++}$gluonium, namely radiative quarkonium decays including charmonium. The second consequence is that there is enough phase-space for $0^{--}$decays with a quite clear experimental signature. We discuss both the issues in section 4 .

\section{Oddballs in medium}

Before addressing the phenomenology of the lightest oddball, it is interesting to use the same holographic machinery to investigate other aspects of this gluonic state, namely its features in a thermalized and dense hadronic medium. The aim is to make a comparison with the conventional light vector meson and with the scalar glueball, inferring information on the stability properties of the oddball against thermal and density fluctuations. In the holographic approach, the inclusion of matter effects is affordable using appropriate bulk geometries [12]. For definiteness, we consider the SW model for which many results concerning other hadrons are available for the comparison [33, 34].

To investigate in-medium effects on the oddball spectrum, we use the $5 D$ ReissnerNordström AdS metric (AdS/RN). The issue of which phase of QCD is described by this bulk geometry is deferred to the end of this section; for the time being, we consider the possibility of describing a stable or a metastable phase [35].

The AdS/RN geometry is defined by the line element

$$
d s_{(R N)}^{2}=g_{(R N)}^{M N} d x_{M} d x_{N}=\frac{1}{z^{2}}\left(f(z) d x_{0}^{2}-d \vec{x}^{2}-\frac{d z^{2}}{f(z)}\right)
$$


with the function $f(z)$ given by

$$
f(z)=1-\left(\frac{1}{z_{h}^{4}}+q^{2} z_{h}^{2}\right) z^{4}+q^{2} z^{6}
$$

At $q=0$ the geometry (3.1), (3.2) reduces to the AdS/black-hole metric. $z_{h}$ is the position of the outer horizon of the black-hole, the lowest value of the coordinate $z$ satisfying the condition $f\left(z_{h}\right)=0$. Defining $Q=q z_{h}^{3}$ and imposing the condition $0 \leqslant Q \leqslant \sqrt{2}$, the black-hole temperature is

$$
T=\frac{1}{4 \pi}\left|\frac{d f}{d z}\right|_{z=z_{h}}=\frac{1}{\pi z_{h}}\left(1-\frac{Q^{2}}{2}\right) .
$$

In (3.2) $q$ is the charge of the black-hole, which can be holographically related to the quark chemical potential $\mu$. In the QCD generating functional, $\mu$ multiplies the quark number operator $O_{q}(x)=q^{\dagger}(x) q(x)$. Invoking the gauge/gravity correspondence, the coefficient $\mu$ can be considered as the source of the bulk field associated to $O_{q}(x)$, the time component of a U(1) gauge field $A_{M}(x, z)$. The AdS/RN metric results from the gravitational interaction of this U(1) field. Within the SW model, we make use of the AdS/RN geometry together with the background dilaton characterizing the model.

To fulfil rotational invariance, the $\mathrm{U}(1)$ field $A_{M}$ has components $A_{i}=0$ for $i=1,2,3, z$ , while the component $A_{0}$ has the expansion, for $z \rightarrow 0$,

$$
A_{0}(z)=\mu-\kappa q z^{2} .
$$

The condition that $A_{0}$ vanishes at the horizon, $A_{0}\left(z_{h}\right)=0$, provides a linear relation between $\mu$ and $q$ (or $\mu$ and $Q$ ),

$$
\mu=\kappa \frac{Q}{z_{h}}
$$

in terms of a dimensionless parameter $\kappa$ that can be determined from various observables [36]. In the following we set $\kappa=1$, giving the quark chemical potential up to a numerical factor. We also set the dilaton parameter $c=1$ and the dimensionful quantities in units of such a scale.

The equation for the bulk-to-boundary propagator $\widetilde{K}(p, z)$, obtained from the action (2.5) with AdS/RN background geometry, reads:

$$
\partial_{z}\left[\frac{e^{-\phi(z)}}{z^{3}} f(z) \partial_{z} \widetilde{K}(p, z)\right]+\frac{e^{-\phi(z)}}{z^{3}}\left[\frac{p_{0}^{2}}{f(z)}-\vec{p}^{2}\right] \widetilde{K}(p, z)-\frac{M_{5}^{2} e^{-\phi(z)}}{z^{5}} \widetilde{K}(p, z)=0 .
$$

In the frame with $\vec{p}=0$, defining $\omega^{2}=p_{0}^{2}$ and using the variable $u=z / z_{h}$, we compute the solution of (3.6) with the boundary conditions

$$
\begin{array}{rlrl}
\widetilde{K}\left(\omega^{2}, u\right) & \sim \frac{1}{z_{h}^{4} u^{4}} & (u \rightarrow 0) \\
\widetilde{K}\left(\omega^{2}, u\right) & \sim(1-u)^{-i \frac{\sqrt{\omega^{2} z_{h}^{2}}}{4-2 Q^{2}}} & & (u \rightarrow 1) .
\end{array}
$$




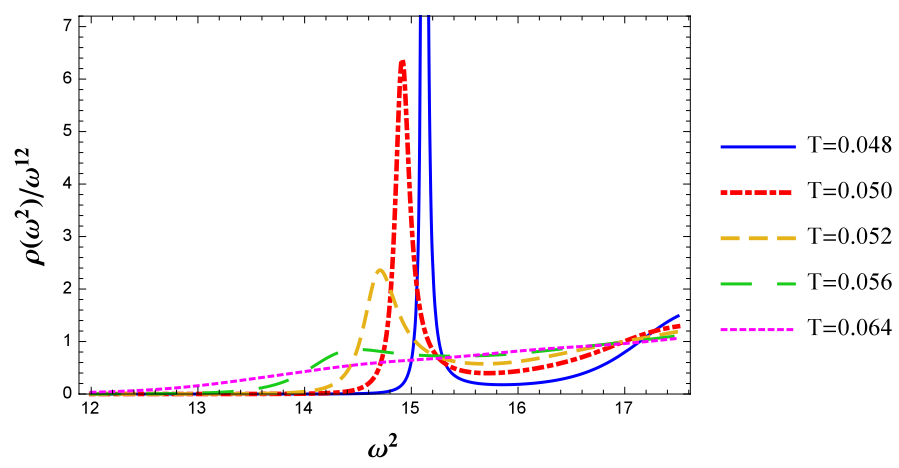

Figure 2. Spectral function for the $J^{\mathrm{PC}}=0^{--}$oddball in the SW model with the metric (3.1)(3.2), at $\mu=0$ and for several values of $T$. In the plotted function $\rho\left(\omega^{2}\right) /\left(\omega^{2}\right)^{6}$ the constant $10^{-8} k /\left(2 z_{h}^{8}\right)$ has been factorized out. The dimensionful quantities are in units of the scale $c$.

The latter condition selects the in-falling solution near the horizon. Hence, the retarded Green's function is worked out [37-39],

$$
\Pi^{R}\left(\omega^{2}\right)=\frac{2}{k}\left[\frac{e^{-\phi(z)}}{z^{3}} f(z) \widetilde{K}\left(\omega^{2}, z\right) \partial_{z} \widetilde{K}\left(\omega^{2}, z\right)\right]_{z \rightarrow 0},
$$

and the spectral function $\rho\left(\omega^{2}\right)=\Im\left(\Pi^{R}\left(\omega^{2}\right)\right)$ is determined in ranges of temperature and chemical potential.

At $\mu=0$, the result in the range of $\omega^{2}$ corresponding to the lightest resonance is depicted in figure 2 , where we plot $\rho\left(\omega^{2}\right) /\left(\omega^{2}\right)^{6}$ (factorizing the constant $10^{-8} k /\left(2 z_{h}^{8}\right)$ ) to account for the $\rho$ large- $\omega^{2}$ dependence. At small $T$ the spectral function displays a narrow resonance, with vanishing width for $T \rightarrow 0$. As $T$ increases changing the bulk geometry, the peak moves towards smaller values of $\omega^{2}$, accompanied by a broadening of the line shape: the thermal effects on the gluonium reduce the mass and make the state unstable. At some value of $T$ the peak disappears from the spectral function, indicating the in-medium melting of the state.

Also at finite $\mu$ the peaks of the spectral function broaden and move towards smaller values of $\omega^{2}$ as $T$ increases, up to a point where no peak can be distinguished. The same behavior is observed at fixed temperature, increasing the chemical potential. The broadening is a signal that, as the temperature or the quark chemical potential increases, the states become unstable, getting a finite width (a quantum-mechanical argument for such a behavior is in [34]). The results are collected in figure 3 for two values of $\mu$ and $T$.

To quantitatively extract the temperature and chemical potential dependence of the lightest oddball mass, we fit the peak in the spectral function $\rho\left(\omega^{2}\right)$ using a Breit-Wignerlike expression $[33,40]$ :

$$
\rho_{B W}\left(\omega^{2}\right)=\frac{a\left(\omega^{2}\right)^{b} m \Gamma}{\left(\omega^{2}-m^{2}\right)^{2}+m^{2} \Gamma^{2}},
$$

obtaining the mass $m$ and width $\Gamma$ of the state ( $a$ and $b$ are parameters in the fit). The melting temperature and chemical potential are obtained looking at the values of $T, \mu$ where the peak in the spectral function is reduced by a factor (we choose 20) with respect 

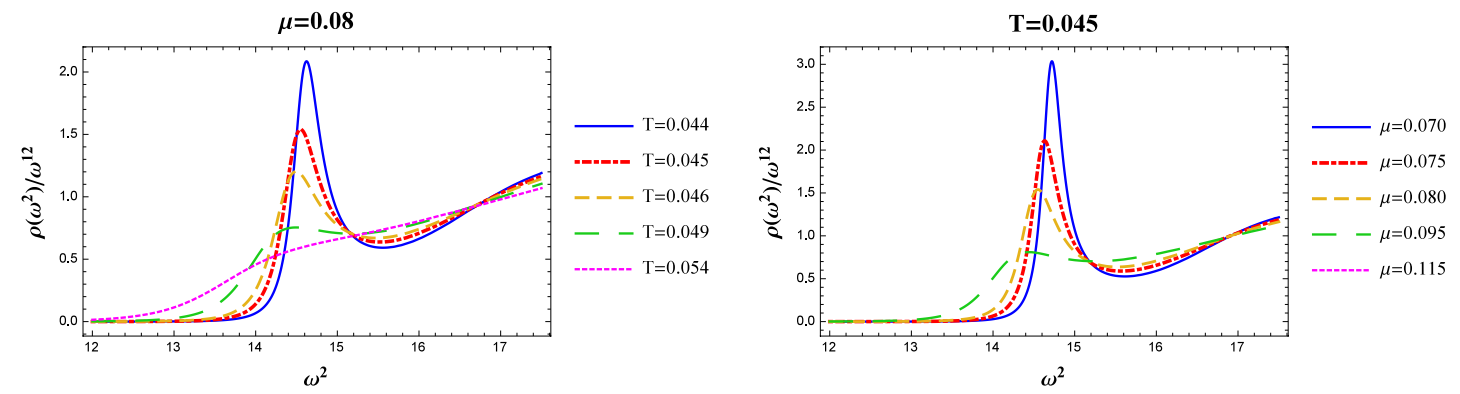

Figure 3. Spectral function $\rho\left(\omega^{2}\right) /\left(\omega^{2}\right)^{6}$ at fixed $\mu=0.08$ for different values of the temperature (left), and at fixed $T=0.045$ for different values of the chemical potential (right). In the plotted function the constant $10^{-8} k /\left(2 z_{h}^{8}\right)$ has been factorized out. The dimensionful quantities are in units of $c$.
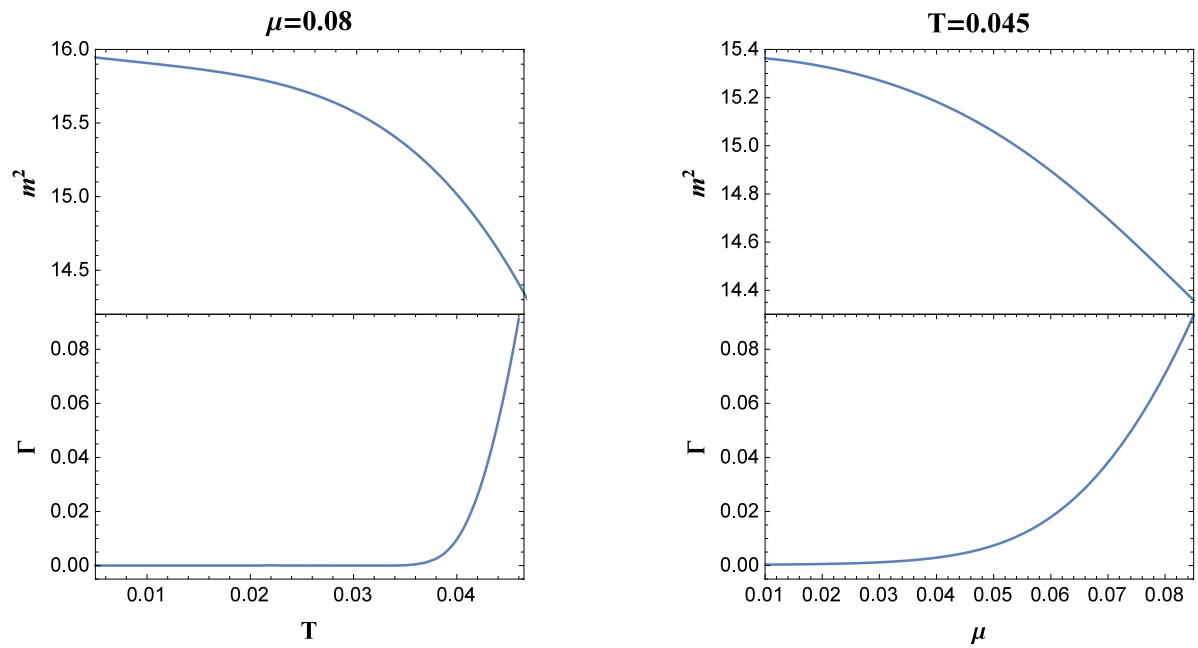

Figure 4. $m^{2}$ (up) and width (down) of the lightest $J^{\mathrm{PC}}=0^{--}$glueball, at fixed $\mu=0.08$ for different values of temperature (left) and at fixed $T=0.045$ for different values of chemical potential (right), in the AdS/RN SW model. The dimensionful quantities are in units of $c$.

to the point where the line-shape starts broadening. The results for two values of $T$ and $\mu$ are shown in figure 4, and a synopsis of the $T-\mu$ dependence is presented in figure 5 .

One can now make a comparison with other hadrons. Considering light vector and scalar $\bar{q} q$ mesons, the lightest scalar glueball and hybrid mesons, the values of $T$ and $\mu$ where the peak of the lowest lying oddball disappears from the spectral function are by far smaller [26, 34]. This can be interpreted as an indication of a higher sensitivity of this hadron to thermal and matter effects at $T \neq 0$ and $\mu \neq 0$, and that the state is less stable than other conventional hadrons.

In the above discussion, we have assumed the AdS/RN geometry as suitable to formulate a QCD dual regardless of the values of $T$ and $\mu$. However, with this geometry it is known that duality holds above a line in the $T-\mu$ plane, where the deconfined phase is realized; at small $T$ and $\mu$ AdS/RN represents a metastable phase, the confining phase being described, e.g. at $\mu=0$, by thermal-AdS. The transition between the two phases is holo- 

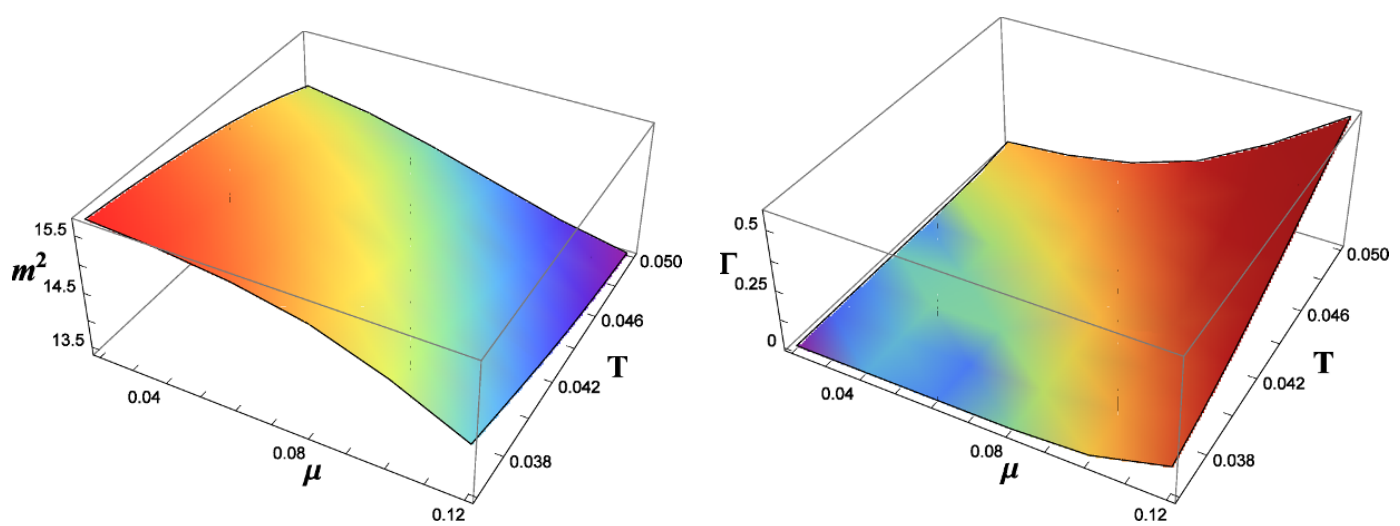

Figure 5. $m^{2}$ and width $\Gamma$ of the lightest $J^{\mathrm{PC}}=0^{--}$glueball, computed in a range of temperature $T$ and chemical potential $\mu$, in the SW model with AdS/RN metric. The dimensionful quantities are in units of the scale in the model, the parameter $c$.

graphically represented by a Hawking-Page transition [35]. This can be seen considering in greater detail the limit $T \rightarrow 0$ with finite chemical potential $\mu$.

In the AdS/Reissner-Nordström model the limit $T \rightarrow 0, \mu \neq 0$ corresponds to $Q^{2} \rightarrow 2$ (while $z_{h} \rightarrow \infty$ corresponds to $T=0$ and $\mu=0$ ). This is the case of an extremal black hole with coinciding outer and inner horizon. The black hole function has a double zero in $z=z_{h}$ :

$$
f(z)=\left(1-\frac{z}{z_{h}}\right)^{2}\left(1+\frac{z}{z_{h}}\right)^{2}\left(1+2 \frac{z^{2}}{z_{h}^{2}}\right) .
$$

Moreover, at $T=0$ the geometry has a horizon and a non-vanishing entropy, a known feature of models based on the $\mathrm{RN}$ metric and used in the framework of the emergent quantum criticality. Other consequences are in the determination of the spectral functions, where the behavior of the solution of the equation of motion near the horizon is needed. For $Q^{2}=2$ the asymptotic solution contains divergent terms proportional to $p_{0}^{2}$, which hinder the selection of the in-falling condition. In studies of, e.g., transport coefficients at $T=0$ and $\mu \neq 0$, the condition $\vec{p} \neq 0$ together with the limit $p_{0} \rightarrow 0$ avoids divergences in the correlation functions [41].

It has been proposed to study the points at $T=0, \mu \neq 0$ considering a model having the function $f(z)$ in the geometry given by [36]

$$
f(z)=1+q^{2} z^{6}
$$

and the time-component $A_{0}(z)$ in (3.4). The metric (3.1), (3.11) is solution of the Einstein equations, with the condition $f\left(z_{h}\right)=0$ replaced by putting to zero the coefficient of $z^{4}$. While in the HW model the vanishing $A_{0}\left(z_{0}\right)=0$ at the IR brane provides a relation between $\mu$ and $q$, in the SW model, a linear relation between $\mu$ and $q$ can be assumed, with the coefficient fixed computing the boundary action [42]; the dilaton term in the action allows to cure the divergence of $f$ and $A_{0}$ at large $z$ (naked singularity). As in the thermal AdS model, the temperature can also be implemented using a periodic Euclidean time coordinate. The obtained geometry (thermal charged AdS - tcAdS) is proposed as 


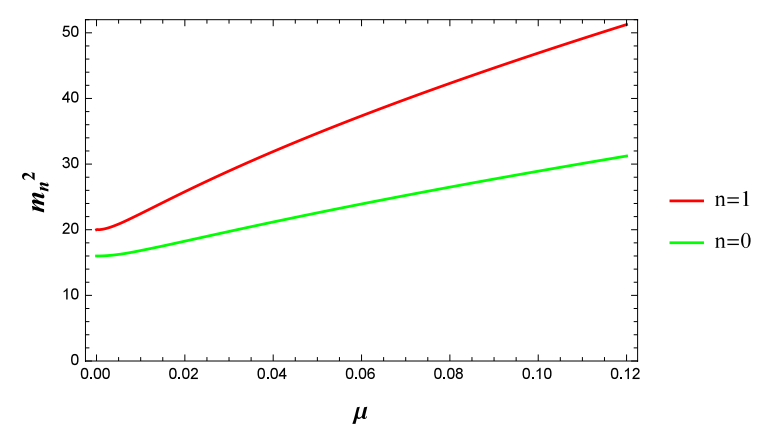

Figure 6. $m^{2}$ vs $\mu$ at $T=0$ for the first two states of the $J^{\mathrm{PC}}=0^{--}$gluonium, in the SW model with geometry (3.1), (3.11). The dimensionful quantities are in units of the scale $c$.

a dual of the confined phase of QCD at small temperature and chemical potential, while the AdS/Reissner-Nordström model describes the deconfined phase, with a Hawking-Page transition between the two phases [36].

Using the geometry (3.1), (3.11) at $T=0$, the two lightest gluonium states have mass as depicted in figure 6 , and at small $T$ the results remain unaffected. The difference with respect to the AdS/RN model is the increasing behaviour vs $\mu$, a confirmation that the two models describe different phases of QCD.

The conclusion of the analysis is that, using AdS/RN, the $0^{--}$oddball is more sensitive to matter effects than all other hadrons studied in the same framework, including the $0^{++}$ glueball and the $1^{+-}$hybrid $[26,34]$. On the other hand, with the tcAdS geometry a peculiar $\mu$-dependence of the hadron mass is found.

\section{$4 \quad J^{\mathrm{PC}}=0^{--}$glueball phenomenology}

In our calculations the lowest gluonium state with $J^{\mathrm{PC}}=0^{--}$is quite light. On the basis of this result, we select suitable processes for the production and the identification of this state. ${ }^{2}$ Our guidelines are the quantum number selection rules, since the relevant hadronic couplings cannot be computed in the models we are using here. For definiteness, we consider the lightest oddball with mass $m_{0^{--}}=2.8 \mathrm{GeV}$.

Production modes in radiative and hadronic two-body transitions, occurring in different waves, are collected in table 2 . Spin $1^{++}$charmonium and bottomonium states decay radiatively in the $0^{--}$oddball in $\mathrm{S}$ - and $\mathrm{D}$-wave, while $2^{++}$states decay in $\mathrm{D}$-wave. The suppression $\frac{\Gamma\left(\chi_{c 1}(1 P) \rightarrow \gamma G\left(0^{--}\right)\right)}{\Gamma\left(J / \psi \rightarrow \gamma G\left(0^{++}\right)\right)} \simeq \alpha_{s}\left(m_{c}\right)$ is expected, while radiative processes in bottomonium, which are phase-space favored, are suppressed by $\left(e_{b} / e_{c}\right)^{2}$ with respect to the corresponding charmonium rates. The hadronic decay mode $X(3872) \rightarrow \omega G\left(0^{--}\right)$is at the limit of the phase space, and the bottomonium modes $\chi_{b 1}(n P) \rightarrow \omega G\left(0^{--}\right)$are allowed. Another interesting process involves the isoscalar scalar $\pi \pi$ configuration in the final state, namely $h_{c}(1 P) \rightarrow \pi^{+} \pi^{-} G\left(0^{--}\right)$, together with the bottomonium counterpart, the $h_{b}(1 P) \rightarrow f_{0}(980) G\left(0^{--}\right)$and $h_{b}(2 P) \rightarrow f_{0}(980) G\left(0^{--}\right)$transitions. Other charmonium decays in P-wave, namely $\psi(n S) \rightarrow G\left(0^{--}\right) \chi_{c 0}(1 P)$ and $\chi_{c 1}(n P) \rightarrow G\left(0^{--}\right) J / \psi$ (with the

\footnotetext{
${ }^{2} \mathrm{~A}$ few modes are mentioned in ref. [7].
} 


\begin{tabular}{|c|c|}
\hline radiative transition & \\
\hline$\chi_{c 1}(3510) \rightarrow \gamma G\left(0^{--}\right)$ & $\chi_{b 1}(9892) \rightarrow \gamma G\left(0^{--}\right)$ \\
$X(3872) \rightarrow \gamma G\left(0^{--}\right)$ & $\chi_{b 1}(10255) \rightarrow \gamma G\left(0^{--}\right)$ \\
$\chi_{c 2}(3556) \rightarrow \gamma G\left(0^{--}\right)$ & $\chi_{b 2}(9912) \rightarrow \gamma G\left(0^{--}\right)$ \\
$\chi_{c 2}(3927) \rightarrow \gamma G\left(0^{--}\right)$ & $\chi_{b 2}(10269) \rightarrow \gamma G\left(0^{--}\right)$ \\
\hline hadronic transition & \\
\hline$X(3872) \rightarrow \omega G\left(0^{--}\right)$ & $\chi_{b 1}(10255) \rightarrow(\omega, \phi, J / \Psi) G\left(0^{--}\right)$ \\
& $\Upsilon(n S) \rightarrow\left(f_{1}(1285), \chi_{c 1}, X(3872)\right) G\left(0^{--}\right)$ \\
$h_{c}(3525) \rightarrow \pi \pi(I=0) G\left(0^{--}\right)$ & $h_{b}(9899) \rightarrow f_{0}(980) G\left(0^{--}\right)$ \\
& $h_{b}(10260) \rightarrow f_{0}(980) G\left(0^{--}\right)$ \\
& $h_{b}(9899) \rightarrow G\left(0^{++}\right) G\left(0^{--}\right)$ \\
& $h_{b}(10260) \rightarrow G\left(0^{++}\right) G\left(0^{--}\right)$ \\
\hline
\end{tabular}

Table 2. Production modes of the $J^{\mathrm{PC}}=0^{--}$glueball, for $m_{0^{--}}=2.8 \mathrm{GeV}$.

\begin{tabular}{|l|}
\hline mode \\
\hline$G\left(0^{--}\right) \rightarrow \gamma f_{1}(1285)$ \\
$G\left(0^{--}\right) \rightarrow \omega f_{1}(1285)$ \\
$G\left(0^{--}\right) \rightarrow \rho a_{1}(1260) \quad(I=0)$ \\
$G\left(0^{--}\right) \rightarrow h_{1}(1270) f_{0}(980)$ \\
$G\left(0^{--}\right) \rightarrow \rho \pi(I=0)$ \\
$G\left(0^{--}\right) \rightarrow K^{*} K(I=0)$ \\
$G\left(0^{--}\right) \rightarrow\left(\eta, \eta^{\prime}\right)(\omega, \phi)$ \\
\hline
\end{tabular}

Table 3. Decay modes of the $J^{\mathrm{PC}}=0^{--}$glueball, $m_{0^{--}}=2.8 \mathrm{GeV}$.

corresponding bottomonium transitions), are only possible for very heavy $\bar{c} c(\bar{b} b)$ decaying states. We remark the P-wave two-body $h_{b}(n P)$ decays in $J^{\mathrm{PC}}=0^{++}$scalar glueball and $J^{\mathrm{PC}}=0^{--}$oddball, which are very peculiar modes for the exclusive gluonium production.

Decay modes of the $J^{\mathrm{PC}}=0^{--}$glueball are listed in table 3 . In addition to the modes involving the axial $I=0 f_{1}(1285)$ meson, it is worth mentioning the full set of $P$-wave decays, among which there is $\rho \pi(I=0)$. The couplings governing the various modes cannot be computed in the framework discussed here, and require specific calculations deferred to a dedicated study. 


\section{Conclusions}

Our main result is that the lowest-lying $J^{\mathrm{PC}}=0^{--}$glueball, examined in different bottomup holographic models of QCD, is lighter than envisaged by other approaches. This opens interesting possibilities for the experimental search of this unconventional hadron. We have also investigated the in-medium effects, obtaining that using AdS/RN the $0^{--}$oddball is more sensitive to matter effects than all other hadrons studied in the same framework. On the other hand, with the tcAdS geometry a peculiar $\mu$-dependence of the hadron mass is found. Several production and decay modes can be exploited for the search of this elusive gluonium resonance.

As a final remark, we find inspiring that the lowest mass we have obtained using the SW model is close to the $D^{0}$ mass, in view of the dominance observed in $D^{0} \rightarrow \pi^{+} \pi^{-} \pi^{0}$ of an exotic $J^{\mathrm{PC}}=0^{--}$isoscalar state. It is worth reconsidering this issue in a dedicated study.

\section{Acknowledgments}

We thank Fulvia De Fazio and Stefano Nicotri for discussions.

Open Access. This article is distributed under the terms of the Creative Commons Attribution License (CC-BY 4.0), which permits any use, distribution and reproduction in any medium, provided the original author(s) and source are credited.

\section{References}

[1] R.L. Jaffe and K. Johnson, Unconventional states of confined quarks and gluons, Phys. Lett. B 60 (1976) 201 [INSPIRE].

[2] W. Ochs, The status of glueballs, J. Phys. G 40 (2013) 043001 [arXiv:1301.5183] [INSPIRE].

[3] H.-Y. Cheng, C.-K. Chua and K.-F. Liu, Revisiting scalar glueballs, arXiv:1503.06827 [INSPIRE].

[4] M. Chanowitz, Chiral suppression of scalar glueball decay, Phys. Rev. Lett. 95 (2005) 172001 [hep-ph/0506125] [INSPIRE].

[5] N. Isgur and J.E. Paton, A flux tube model for hadrons in QCD, Phys. Rev. D 31 (1985) 2910 [INSPIRE].

[6] E. Gregory et al., Towards the glueball spectrum from unquenched lattice QCD, JHEP 10 (2012) 170 [arXiv:1208.1858] [INSPIRE].

[7] C.-F. Qiao and L. Tang, Finding the $0^{--}$glueball, Phys. Rev. Lett. 113 (2014) 221601 [arXiv: 1408.3995] [INSPIRE].

[8] S. Malde et al., First determination of the CP content of $D \rightarrow \pi^{+} \pi^{-} \pi^{+} \pi^{-}$and updated determination of the CP contents of $D \rightarrow \pi^{+} \pi^{-} \pi^{0}$ and $D \rightarrow K^{+} K^{-} \pi^{0}$, Phys. Lett. B 747 (2015) 9 [arXiv: 1504.05878] [INSPIRE].

[9] J.M. Maldacena, The large- $N$ limit of superconformal field theories and supergravity, Int. J. Theor. Phys. 38 (1999) 1113 [hep-th/9711200] [INSPIRE]. 
[10] E. Witten, Anti-de Sitter space and holography, Adv. Theor. Math. Phys. 2 (1998) 253 [hep-th/9802150] [INSPIRE].

[11] S.S. Gubser, I.R. Klebanov and A.M. Polyakov, Gauge theory correlators from noncritical string theory, Phys. Lett. B 428 (1998) 105 [hep-th/9802109] [INSPIRE].

[12] M. Ammon and J. Erdmenger, Gauge/gravity duality, Cambridge University Press, Cambridge U.K. (2015).

[13] D.J. Gross and H. Ooguri, Aspects of large- $N$ gauge theory dynamics as seen by string theory, Phys. Rev. D 58 (1998) 106002 [hep-th/9805129] [INSPIRE].

[14] C. Csáki, H. Ooguri, Y. Oz and J. Terning, Glueball mass spectrum from supergravity, JHEP 01 (1999) 017 [hep-th/9806021] [INSPIRE].

[15] A. Hashimoto and Y. Oz, Aspects of QCD dynamics from string theory, Nucl. Phys. B 548 (1999) 167 [hep-th/9809106] [INSPIRE].

[16] N.R. Constable and R.C. Myers, Spin two glueballs, positive energy theorems and the AdS/CFT correspondence, JHEP 10 (1999) 037 [hep-th/9908175] [INSPIRE].

[17] R.C. Brower, S.D. Mathur and C.-I. Tan, Glueball spectrum for QCD from AdS supergravity duality, Nucl. Phys. B 587 (2000) 249 [hep-th/0003115] [INSPIRE].

[18] D. Elander, A.F. Faedo, C. Hoyos, D. Mateos and M. Piai, Multiscale confining dynamics from holographic RG flows, JHEP 05 (2014) 003 [arXiv: 1312.7160] [INSPIRE].

[19] K. Hashimoto, C.-I. Tan and S. Terashima, Glueball decay in holographic QCD, Phys. Rev. D 77 (2008) 086001 [arXiv: 0709.2208] [INSPIRE].

[20] F. Brünner, D. Parganlija and A. Rebhan, Glueball decay rates in the Witten-Sakai-Sugimoto model, Phys. Rev. D 91 (2015) 106002 [arXiv:1501.07906] [INSPIRE].

[21] J. Erlich, E. Katz, D.T. Son and M.A. Stephanov, QCD and a holographic model of hadrons, Phys. Rev. Lett. 95 (2005) 261602 [hep-ph/0501128] [inSPIRE].

[22] L. Da Rold and A. Pomarol, Chiral symmetry breaking from five dimensional spaces, Nucl. Phys. B 721 (2005) 79 [hep-ph/0501218] [INSPIRE].

[23] A. Karch, E. Katz, D.T. Son and M.A. Stephanov, Linear confinement and AdS/QCD, Phys. Rev. D 74 (2006) 015005 [hep-ph/0602229] [INSPIRE].

[24] P. Colangelo, F. De Fazio, F. Giannuzzi, F. Jugeau and S. Nicotri, Light scalar mesons in the soft-wall model of AdS/QCD, Phys. Rev. D 78 (2008) 055009 [arXiv:0807.1054] [INSPIRE].

[25] S.J. Brodsky, G.F. de Teramond, H.G. Dosch and J. Erlich, Light-front holographic QCD and emerging confinement, Phys. Rept. 584 (2015) 1 [arXiv: 1407.8131] [INSPIRE].

[26] L. Bellantuono, P. Colangelo and F. Giannuzzi, Exotic $J^{P C}=1^{-+}$mesons in a holographic model of QCD, Eur. Phys. J. C 74 (2014) 2830 [arXiv:1402.5308] [INSPIRE].

[27] H. Boschi-Filho and N.R.F. Braga, QCD/string holographic mapping and glueball mass spectrum, Eur. Phys. J. C 32 (2004) 529 [hep-th/0209080] [INSPIRE].

[28] P. Colangelo, F. De Fazio, F. Jugeau and S. Nicotri, On the light glueball spectrum in a holographic description of QCD, Phys. Lett. B 652 (2007) 73 [hep-ph/0703316] [INSPIRE].

[29] H. Forkel, Holographic glueball structure, Phys. Rev. D 78 (2008) 025001 [arXiv:0711.1179] [INSPIRE]. 
[30] P. Colangelo, F. De Fazio, F. Jugeau and S. Nicotri, Investigating AdS/QCD duality through scalar glueball correlators, Int. J. Mod. Phys. A 24 (2009) 4177 [arXiv:0711.4747] [INSPIRE].

[31] D. Li, S. He, M. Huang and Q.-S. Yan, Thermodynamics of deformed AdS $S_{5}$ model with a positive/negative quadratic correction in graviton-dilaton system, JHEP 09 (2011) 041 [arXiv: 1103.5389] [INSPIRE].

[32] D. Li and M. Huang, Dynamical holographic QCD model for glueball and light meson spectra, JHEP 11 (2013) 088 [arXiv: 1303.6929] [INSPIRE].

[33] P. Colangelo, F. Giannuzzi and S. Nicotri, Holographic approach to finite temperature QCD: the case of scalar glueballs and scalar mesons, Phys. Rev. D 80 (2009) 094019 [arXiv: 0909.1534] [INSPIRE].

[34] P. Colangelo, F. Giannuzzi and S. Nicotri, In-medium hadronic spectral functions through the soft-wall holographic model of QCD, JHEP 05 (2012) 076 [arXiv:1201.1564] [INSPIRE].

[35] C.P. Herzog, A holographic prediction of the deconfinement temperature, Phys. Rev. Lett. 98 (2007) 091601 [hep-th/0608151] [INSPIRE].

[36] B.-H. Lee, C. Park and S.-J. Sin, A dual geometry of the hadron in dense matter, JHEP 07 (2009) 087 [arXiv:0905.2800] [InSPIRE].

[37] D.T. Son and A.O. Starinets, Minkowski space correlators in AdS/CFT correspondence: recipe and applications, JHEP 09 (2002) 042 [hep-th/0205051] [INSPIRE].

[38] G. Policastro, D.T. Son and A.O. Starinets, From AdS/CFT correspondence to hydrodynamics, JHEP 09 (2002) 043 [hep-th/0205052] [INSPIRE].

[39] D. Teaney, Finite temperature spectral densities of momentum and $R$-charge correlators in $N=4$ Yang-Mills theory, Phys. Rev. D 74 (2006) 045025 [hep-ph/0602044] [InSPIRE].

[40] M. Fujita, K. Fukushima, T. Misumi and M. Murata, Finite-temperature spectral function of the vector mesons in an AdS/QCD model, Phys. Rev. D 80 (2009) 035001 [arXiv:0903.2316] [INSPIRE].

[41] M. Edalati, J.I. Jottar and R.G. Leigh, Transport coefficients at zero temperature from extremal black holes, JHEP 01 (2010) 018 [arXiv:0910.0645] [INSPIRE].

[42] C. Park, D.-Y. Gwak, B.-H. Lee, Y. Ko and S. Shin, The soft wall model in the hadronic medium, Phys. Rev. D 84 (2011) 046007 [arXiv: 1104.4182] [INSPIRE]. 